\title{
Survey on Robust and Reversible Watermarking for Relational Data
}

\author{
Udaya Rani, $\mathrm{PhD}$ \\ Sr Associate Professor \\ Computer Science and \\ Engineering \\ RITM, Bangalore
}

\author{
Shreedevi B. Choudri \\ Master of Technology in \\ Computer Science and \\ Engineering \\ RITM, Bangalore
}

\author{
Vinayak Murthy \\ Assistant director research and \\ innovation \\ RITM, Bangalore
}

\begin{abstract}
In the course of recent years, reversible watermarking systems for social databases have been proposed to give assurance of possession rights, information treating, and information trustworthiness. Every now and again, these strategies make certain fashioned information recuperation from knowledge of the watermarks, though irreversible watermarking plans best safeguard possession rights. At the point when possession rights are upheld utilizing watermarking, the basic information experiences specific alteration; as a consequence of which, the information quality gets traded off. Reversible watermarking is utilized to guarantee information quality alongside information recuperation. Nevertheless, such approaches are in general now not strong towards malicious assaults and what's more, don't supply any component to specifically watermark a one of a kind quality by means of considering its position in capacities disclosure.
\end{abstract}

\section{General Terms}

Genetic algorithm, watermarking insertion technique

\section{Keywords}

Reversible Watermarking, Genetic Algorithm, Robustness

\section{INTRODUCTION}

In today's world data is generated due to the high use of cloud computing and internet. Usually data stored in different format like video, audio, images. Watermarking strategies have generally been utilized to ensure security regarding possession safeguard and sealing for information designs and also watermarking technology used to protect data from hackers. The watermarking program introduces small error into the object being watermarked. The watermarking programming brings little blunders into the item being watermarked. These deliberate mistakes are assembled marks and every one of the imprints constitutes the watermark. The imprints must not significantly affect the helpfulness of the information and they ought to be put in a manner that a malevolent client can't pulverize them without making the information less valuable. A type of computerized watermarking procedures are getting utilized over the way of the last couple of numerous years for proprietorship shield of advanced media reminiscent of photographs, sound, video [13], and normal dialect handling application [4]. With the expanding notoriety of

progressed for social databases [5-7]. Information proprietors permit their information to be gotten to and utilized remotely; along these lines Presenting them to risk of information burglary. In spite of the fact that watermarking innovation is supportive in demonstrating proprietorship through distinguishing proof of information theft, the drawback is that it presents changeless alterations in the information which are irreversible and causes the coming about watermarked information to end up not quite the same as the unique substance. Subsequently, information investigation and choice making on the contorted rendition of information gets to be incomprehensible.. Digital watermarking manner has been successively applied to preserve the multimedia works and software merchandise. In a similar way, database watermarking has been proposed on massive database safetymanipulate. Reversible watermarking of social databases is a generally new and developing region. It is a decent competitor answer for guaranteeing information proprietorship assurance and its uprightness. Social information have a specific configuration and are not quite the same as that of other advanced information, for example, sound, video, programming, and pictures. The strategies for possession insurance and information recuperation of social information needs to consider new limitations taking into account actualities, for example, (i) a database comprises of tuple/records, which is the place the watermark should be installed; (ii) the requesting of the records in a database connection; and (iii) information operations such as insertion, cancellation, and adjustment that regularly happen in a database. Besides, contingent upon the assortment of data spared inside of the database [8]. Reversible water marking technique it recovers data with ownership protection. Some technique used for ownership protecting such as hash function, fingerprinting [9]. Interactive media watermarking is extremely not quite the same as that used to watermark social databases due to a major distinction in the properties of the information. Interactive media information is exceptionally associated and ceaseless while social information is free and discrete. With the appearance of current copyright insurance and data concealing systems, database watermarking can be utilized to implement possession privileges of social information.. These all technique modify the data very large but they loss actual data quality. To overcome this problem reversible watermarking technique is used.

\section{OVERVIEW DATABASE WATERMARKING}

Now-a-days various data on the web is on hand on the type of databases. So it is very major for the proprietor of the databases point of view that the ownership of the database is maintained at any place it goes[10].There are two forms of database on the groundwork of the attributes they include:

- Numerical Databases

- Non Numerical Databases

Numerical databases include one or more than one attributes which contain numbers. We assume that these numerical databases provide some sort of lenity in a number of than numerical fields so that we can change the attribute worth to 
embed the watermark. Non numerical databases incorporate simplest textual fields which are not dependable to be transformed. Watermarking in such kinds of databases can be achieved in two ways:

\section{1) Distortion Free Watermarking \\ 2) Loose field Watermarking}

In Distortion Free watermarking we extract an watermark from the database and then examine the watermark with the a copy of that database to infer possession Of the database. But in via the database owner. On this variety of watermarking scheme, we change one or a couple of free fields to embed the watermark. Unfastened subject watermarking, we seek for the free fields within the database which is provided.

\section{LITERATURE SURVEY}

In this section of proposal research literature review is presented.

In [11] author proposed the first irreversible water marking technique for the relation database In this technique only numeric attributes is marked and marked attribute can change the some value. In relation only numeric attribute is not be marked.

In [12] author proposed method multiple watermarking for recovering information. It provides two main objectives: identification of ownership proof and recovering information. To resolve conflict between ownership Watermark is embedded using secret key it known only the owner of database

In [13] authors worked on Generic algorithms and difference expansion to improve capacity of watermark and reduce the distortions. GA also called as optimization technique. Distortions are created by the difference expansion it helps the attacker to easy to imagine watermarked attributes. To achieve reversibility in database we use difference expansion watermarking. Attacker s use distortions to easily imagine attributes of the watermark it may be affect watermark detection because DEW not possible to increase watermark capacity without increasing distortions attributes. So that proposed one more technique GADEW. By using the Watermark mean and devotion it's easy to evaluate the attributes in distortions.

Reference [14] defines blind reversible technique for relational database. This method ensures protection of ownership in the field of relational database. This approach ensures the after watermark has been authenticated and detected it recovery $100 \%$ of original database owner of database verify the ownership of database using watermark extraction algorithm. Completely it recovers the original database and also reconstruct embedded watermark by using bit decoding algorithm.

Reference [15] the scheme, proof concept for watermarking it elastic to attacks such insertion, deletion and also alteration. It mainly include the one feature Deductibility i.e. with help of key it can be easy to retrieve the original data. In relational database watermark unnoticeable because proof of the ownership based on secure embed robustly. It mainly involves the encoding and decoding algorithms for relational database but attributes must be numerical in relational database. Here authors using bit encoding and bit decoding algorithms.

In [17] proposed the PSO and GA. Both PSO and GA based on heuristic population methods. Objective of this paper is comparing both GA AND PSO efficiency and effectiveness by using hypothesis testing approaches. . In single iteration both GA AND PSO move the one element into another element by using implementation of probabilistic and deterministic rules. Now days GA is most popular in industry because of intuitive, it's have high ability to solve nonlinear problems and also implementation is easy. But one main drawback of GA is cost is more. In this paper authors declare the PSO also have the same effectiveness of the GA but evaluations of function is less by implementing hypothesis testing and statistical analysis and also comparison of the performance is implemented by the benchmark test. In [16] worked on detect and characterize the malicious modification to database by using fragile zero watermarking. Earlier watermarking technique for relational databases introduce permanent damage into original content of the database as marked. Damage automatically breakdown the quality of the data and usability of data as relational database is violet. Fragile watermark detect the particular where exactly modification done but not characterize attacks. The proposed delicate plan depends on zero watermarking way to deal with recognize malignant adjustments made to a database connection. In zero watermarking, the watermark is produced (built) from the substance of the first information instead of presentation of lasting mutilations as imprints into the information. Proposed scheme solves the conflict between importability and security and also its distortion free.

In [18] authors worked on detect and locate malicious alteration to database categorical attribute by using fragile watermarking. Proposed technique distortions free to cover data but other watermarking techniques automatically introduce the damage to database. First, database relation all tuple divided into groups according to secure parameters. After that each group verification of watermark and watermark embedded done independently and any alteration or modification of data can be locate in some other specific groups.

\section{MAIN OBJECTIVES}

The most important goal of proposed study is to study:

a) watermark accounting so as to encode and interpreting for the part of the considerable number of components in information disclosure

b) Normal information restoration in the presence of energetic malicious attacks.

\section{EXISTING SYSTEM}

Computerized watermarking of media substance is more normally known. Especially picture watermarking-a subsidiary of Steganography is an age-old work on permitting incognito transmission of messages starting with one gathering then onto the next by abusing excess in like manner picture designs. Be that as it may the essential procedure of mixed media watermarking is extremely unique in relation to that used to watermark social databases as a result of a major distinction in the properties of the information.

Mixed media information is exceptionally related and constant while social information is free and discrete. With the appearance of cutting edge copyright assurance and data concealing systems, database watermarking can be utilized to implement proprietorship privileges of social information however a significant disadvantage of these strategies is that they alter the information to a substantial degree which regularly brings about the loss of information quality. 


\section{PROPOSED SYSTEM}

In the proposed procedure we're utilizing Genetic algorithm. Reversible watermarking manner (RRW) proposed to gain a most appropriate resolution that's attainable for the current issue what's more, does not abuse the characterized requirements. An ideal watermark worth is made through the GA and embedded into the chose highlight of the social database in one of this approach that the information satisfying and stay the undamaged. In RRW, shared data is utilized to choose a suitable (applicant) highlight from the database for watermarking. By, existing reversible watermarking methods, don't consider the shared data measure for deciding relative significance of highlights. In RRW, the learning of common data for each competitor highlight is likewise utilized to figure the watermark data. Consequently, it is guaranteed that the information quality won't be influenced. Hence, RRW gives a powerful answer for information recuperation that is off learning quality furthermore A robust regain the first information that is strong contrary to subset adjustment, subset cancellation and subset insertion assaults. The configuration of an astute reversible watermarking process for social information that guarantees

information recovery without trading off learning quality furthermore A robust regain the first information that is strong contrary to subset adjustment, subset cancellation and subset insertion assaults.

\section{Different Types of Attack}

Subset addition assault: in this assault, the attacker tries to insert new tuple into the asset in the hope of destroying the watermark.

Subset erasure assault: In this assault, the aggressor tries to erase the tuple of the advantage in any desire for pulverizing the watermark.

Subset alteration attack: on this assault, the attacker tries to change the values of probably the most tuple to be able to break the watermark.

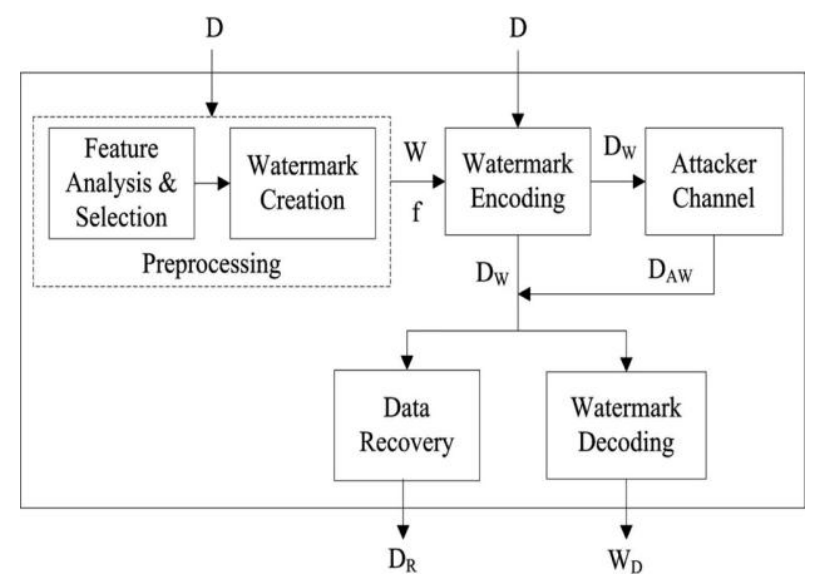

\section{METHOD LOGY}

In the proposed work RRW using Genetic algorithm. GA keeps up a populace of potential arrangement of the competitor issue termed as person. By control of these person through hereditary administrators, for example, determination, mutation, crossover. Execution of GA show in underneath figure 2 .

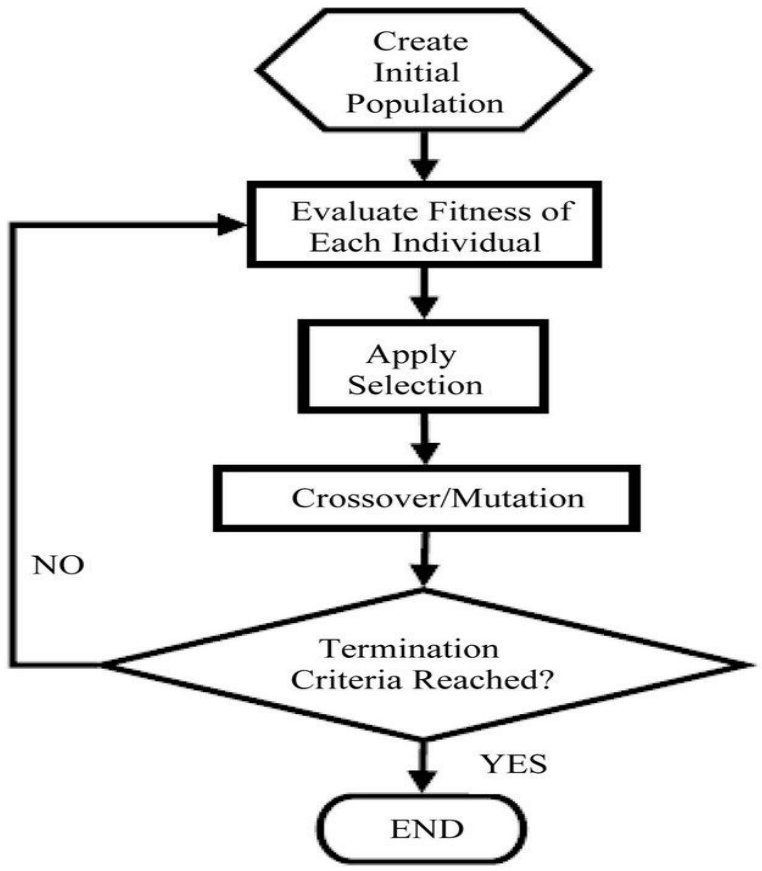

8. CONCLUSIONS

Irreversible watermarking methods roll out improvements in the information to such a degree, to the point that information quality gets traded off. Reversible watermarking methods are utilized to take into account such situations since they can recuperate unique information from watermarked information and guarantee information quality to some degree. Be that as it may, these procedures are definitely not strong against noxious assaults.

\section{REFERENCES}

[1] Cox I, Miller M, Bloom J, Miller M. Digital Watermarking. Morgan Kaufmann: San Francisco, California, 2001.

[2] Wong PW, Memon N. Secret and public key image watermarking schemes for image authentication and ownership

[3] Verification.IEEE Transactions on Image Processing 2001; 10(10): 1593-1601.

[4] Petit colas FA. Watermarking schemes evaluation. IEEE Signal Processing Magazine 2000; 17(5): 58-64.

[5] Brassil J T, Low S, Maxemc huk NF."Copyright protection for the electronic distribution of text documents" .Proceedings of the IEEE 1999; 87(7): $1181-1196$.

[6] Agrawal R, Kiernan J. Watermarking relational databases, Proceedings of the 28th international conference on Very Large Data Bases, VLDB Endowment, Hong Kong, 2002; 155-166.

[7] Sion R, Attala M, Prabhakar S. Right's protection for relational data. IEEE Transactions on Knowledge and Data Engineering 2004; 16(12): 1509-1525.

[8] Sion R, Attala M, Prabhakar S. Right's protection for categorical data. IEEE Transactions on Knowledge and Data Engineering 2005; 17(7): 912-926.

[9] M. Kamran and Zahid Anwar A survey on reversible watermarking techniques for relational databases 2015.

[10] S. Subramanya and B. K. Yi, "Digital rights management," IEEE Potentials, vol. 25, no. 2, pp. 3134, Mar.-Apr. 2006.

[11] Ashu Gupta and Mohd Dawood "Digital and Database Watermarking "2012. 
[12] 11. R. Agrawal and J. Kiernan, "Watermarking relational databases," in Proc. 28th Int. Conf. Very Large Data Bases, 2002, pp. 155-166.

[13] Shampa Chakraverty and Om Prakash Verma "A Robust Multiple Watermarking Technique for Information Recovery" 2014.

[14] K. Jawad and A. Khan, "Genetic algorithm and difference expansion based reversible watermarking for relational databases," J. Syst. Softw.vol. 86, no. 11, pp. 2742-2753, 2013.

[15] Shi-Jinn Horng and Xian Wang "A novel blind reversible method for watermarking relational Databases", 2013.

[16] Loganayaki "Robust Watermarking For Relational Database "International Journal of Communication and
Computer Technologies Volume 01 - No.41, Issue: 05 May 2013.

[17] Aihab Khan1 and Syed Afaq Husain "A Fragile Zero Watermarking Scheme to Detect and Characterize Malicious Modifications in Database Relations" Hindawi Publishing Corporation The Scientific World Journal Volume 2013.

[18] .R. Hassan, B. Cohanim, O. De Weck, and G. Venter, "A comparison of particle swarm optimization and the genetic algorithm," in Proc. 46th AIAA/ASME/ASCE/AHS/ASC , Struct. Dyn. Mater. Conf., 2005, pp. 1-13.

[19] Yingjiu Li and Huiping Guo "Tamper Detection and Localization for Categorical Data Using Fragile Watermarks" 2004. 